2018-3

\title{
Numerical Evaluation of Microwave Thermal Ablation to Treat Small Adrenocortical Masses
}

\author{
G. Ruvio \\ Technological University Dublin \\ J. Eaton-Evans \\ National University of Ireland Galway, Ireland.
}

A. Shahzad

National University of Ireland Galway, Ireland.

See next page for additional authors

Follow this and additional works at: https://arrow.tudublin.ie/nanolart

Part of the Nanotechnology Commons

\section{Recommended Citation}

Ruvio, G. et al. (2018) Numerical Evaluation of Microwave Thermal Ablation to Treat Small Adrenocortical Masses, INTERNATIONAL JOURNAL OF RF AND MICROWAVE COMPUTER-AIDED ENGINEERING, 28 (3):SI 10.1002/mmce.21236 MAR 2018

This Article is brought to you for free and open access by the NanoLab at ARROW@TU Dublin. It has been accepted for inclusion in Articles by an authorized administrator of ARROW@TU Dublin. For more information, please contact arrow.admin@tudublin.ie, aisling.coyne@tudublin.ie,gerard.connolly@tudublin.ie.

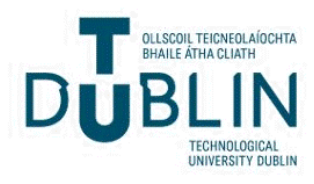


Authors

G. Ruvio, J. Eaton-Evans, A. Shahzad, and M. O'Halloran

This article is available at ARROW@TU Dublin: https://arrow.tudublin.ie/nanolart/72 


\section{Numerical evaluation of microwave thermal ablation to treat small adrenocortical masses}

Article in International Journal of RF and Microwave Computer-Aided Engineering · January 2018

DOI: $10.1002 / m m c e .21236$

CITATIONS

0

4 authors:

\section{Giuseppe Ruvio}

National University of Ireland, Galway

65 PUBLICATIONS 281 CITATIONS

SEE PROFILE

\section{Atif Shahzad}

National University of Ireland, Galway

19 PUBLICATIONS 88 CITATIONS

SEE PROFILE
READS

37

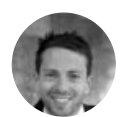

Jimmy Eaton-Evans

National University of Ireland, Galway

28 PUBLICATIONS 89 CITATIONS

SEE PROFILE

Martin O'Halloran

National University of Ireland, Galway

74 PUBLICATIONS 451 CITATIONS

SEE PROFILE

Some of the authors of this publication are also working on these related projects: 


\title{
Numerical evaluation of microwave thermal ablation to treat small adrenocortical masses
}

\author{
G. Ruvio $^{(1,2)}$, J. Eaton-Evans ${ }^{(1)}$, A. Shahzad ${ }^{(1)}$, M. O’Halloran ${ }^{(1,3)}$
}

(1) College of Engineering and Informatics, National University of Ireland Galway, Ireland.

(2) Antenna \& High Frequency Research Centre, Dublin Institute of Technology, Ireland.

(3) School of Medicine, National University of Ireland Galway, Ireland.

\begin{abstract}
In this paper microwave thermal ablation is numerically evaluated in the context of a treatment for Conn's Syndrome. This condition is caused by a benign shallow tumour in the cortex of adrenal gland. The modelling and design of microwave applicator to deliver thermal ablation to the adrenal gland requires accurate tissue characterisation. Measuring the dielectric properties of the constituent tissues in the adrenal gland, i.e. cortex and medulla, enables more accurate numerical modelling for electromagnetic and thermal simulations. This study presents an anatomically and dielectrically realistic numerical model of the adrenal gland, and investigates the feasibility of applying controlled heating to small targets in the adrenal cortex. In addition, the use of dielectric contrast between the fat and the cortex of the adrenal gland to focus the thermal energy in the gland has also been studied. Being conscious of limitations of numerical simulation of complex multiphysics problems like the microwave ablative treatment, calculated results provide a preliminary description of the electromagnetic and thermal phenomena involved.
\end{abstract}

\section{INTRODUCTION}

High blood pressure, also known as hypertension, is a global health problem that accounts for more than 9.4 million deaths every year, and affects over 1 billion people worldwide [1]. Hypertension directly causes approximately $25 \%$ of heart attacks in Europe and, in its progressed form of cardiovascular disease, is responsible for $42 \%$ of all deaths across Europe annually. One of the most common curable cause of secondary hypertension is Primary Aldosteronism (PA, also known as Conn's Syndrome) [2, 3]. PA is thought to account for up to $18 \%$ of all cases of hypertension within the hypertensive population [3, 4]. It is caused by benign, shallow, hormone-eluding adenomas on the cortex of the adrenal gland. A simplified anatomical model of the adrenal gland is 
shown in Fig. 1. Adrenal adenomas can vary in size considerably, but are typically less than $1 \mathrm{~cm}$ in diameter, and are usually identified incidentally during radiologic examinations [5].

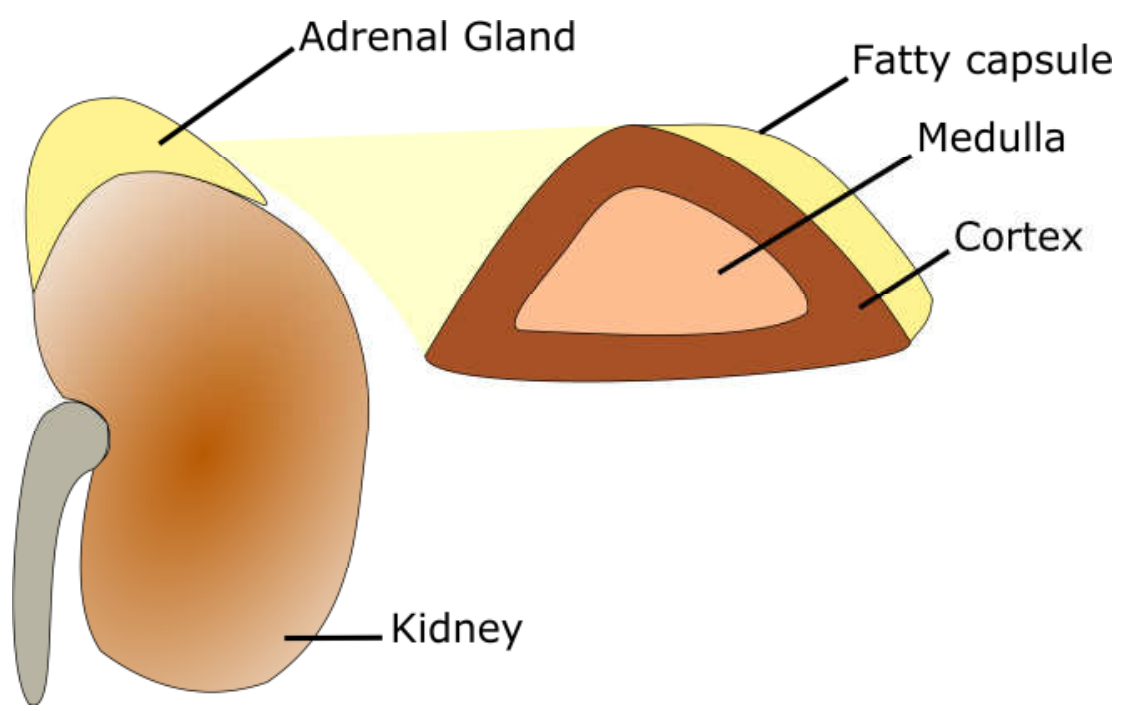

Figure 1: Constituent tissues of the adrenal gland.

PA and associated hypertension can be cured by eliminating these adenomas, however existing treatment options are limited. Currently the most commonly performed treatment for PA is an adrenalectomy, which involves the complete removal of the diseased adrenal gland [3, 6, 7]. This approach has been shown to be effective in treatment of PA where it is confined to one gland [7], with biochemical levels and blood pressure returning to normal [6]. However, PA often reoccurs in the remaining contralateral adrenal gland. In such cases the disease must be managed medically, but available drug treatments are poorly tolerated by patients due to their significant side-effects [7]. These challenges provide a clear motivation for a less invasive treatment that can be used to precisely target the hormone-eluding adenomas in the adrenal gland. It should spare healthy tissue to ensure that the function of the gland is preserved, and eliminate the requirement for surgical resection.

Thermal ablation is accepted as a safe and effective alternative to surgical resection for elimination of lesions in a range of organs including the liver, kidneys and lungs [8]. Early thermal ablation systems used Radio Frequency Ablation (RFA), and were designed primarily for ablation of liver tumours. However, RFA systems have also been used in other clinical applications, such as kidney, lung, bone, and adrenal glands $[9,10,11]$. RFA produces resistive heating by applying electric current in the targeted lesion. The heat is dissipated into the surrounding tissue by thermal 
conduction. This makes it susceptible to heat sinks or changes to tissue impedance which can result in an undesirable elliptical or non-uniform ablation zone [9].

The emergence of Microwave Ablation (MWA) has addressed some of the problems in RFA, providing a means to achieve a faster and more controlled ablation and with less critical dose of anaesthetics $[11,12,13]$. This makes MWA well suited for treatment of lesions in the adrenal gland where a precise ablation zone is required to spare healthy tissue [14].

The rate of heating during MWA is a function of the dielectric properties of the target tissue, which are a function of the water content of the target tissue [12]. The adrenal gland is composed of three principal tissue layers: an outer fatty capsule, the cortex where the hormone-eluding adenomas are located, and the medulla (see Figure 1). Each tissue type in the adrenal gland has different dielectric properties which will affect the rate of heating and the characteristics of the ablation zone. Therefore, the knowledge of accurate dielectric properties and anatomy of the gland is critical in designing a MWA system for treatment of adrenal tumours.

This paper presents an anatomically realistic and dielectrically accurate numerical model of the adrenal gland for MWA at $2.45 \mathrm{GHz}$. The effect of gland structure, ablation power, and therapy duration on the ablation zone is investigated through numerical simulations performed by using the 3-D Computer Simulation Technology Microwave Studio (CST MWS Suite 2017, Darmstadt, Germany). In addition, the feasibility of using the differential in tissue properties to control the ablation zone is investigated. To the best of our knowledge, this is the first numerical evaluation of the microwave ablation in the adrenal gland that models the dielectric properties of fat, cortex and medulla components.

The remainder of this paper is organized as follows: Section 2 describes the numerical model and details the dielectric and thermal properties of the tissues. The multi-physics electromagnetic thermal ablative problem is discussed in Section 3 and conclusions are presented in Section 4. 


\section{NUMERICAL MODELLING}

The adrenal gland represents a complex scenario to model, both in terms of tissue heterogeneity and morphology. Figure 2 shows an anatomical model of a typical adrenal gland site in adult human based on the data in [15].

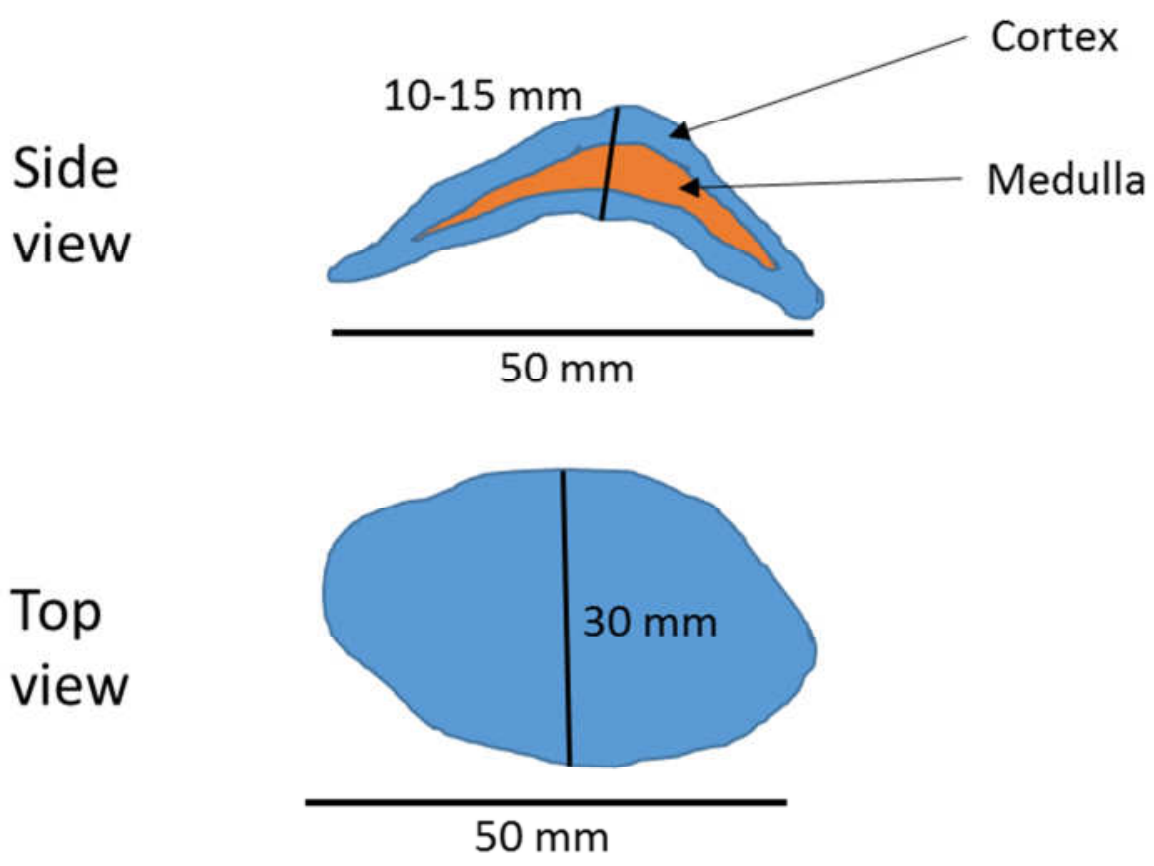

Figure 2: Anatomical model of a typical adrenal gland in adult human

The adrenal gland sits on top of the kidney and it is surrounded by an infiltrated fat capsule. The fat tissue is then covered in a thin film of connective tissue. Accurate modelling of the adrenal site for electromagnetic/thermal multi-physics simulations requires a realistic characterization of each tissue in terms of dielectric and thermal properties at the target frequency and temperature operating range.

A recent study on the dielectric properties of the adrenal gland reported anatomically realistic dielectric model of the gland across the frequency range $0.5-20 \mathrm{GHZ}$ [15]. This study overcomes the issues with incorrect modelling of tissue in most of the recent literature $[17,18]$ where a homogeneous dielectric model was introduced for the adrenal gland. In [16] a slim-form dielectric probe (Keysight 85070E, Santa Rosa, CA, USA) was used for measurements in conjunction with a vector network analyser (Keysight E8362B) to record the reflection coefficient $\left(\mathrm{S}_{11}\right)$ at 101 linearly spaced discrete frequency points over 0.5 to $20 \mathrm{GHz}$. The $S_{11}$ parameters were converted to complex permittivity using Keysight material measurement software suite (Keysight N1500A). 
Dielectric properties are generally expressed in the form of a parametric model. Cole-Cole model is commonly used to represent the frequency dependent permittivity of biological tissues. In the ColeCole mode, the frequency dependent complex permittivity is represented as:

$$
\varepsilon(\omega)=\varepsilon_{\infty}+\sum_{n=1}^{N} \frac{\Delta \varepsilon_{n}}{1+\left(j \omega \tau_{n}\right)^{1-\alpha_{n}}}+\frac{\sigma_{s}}{j \omega \varepsilon_{0}}
$$

where $\varepsilon_{\infty}$ is the permittivity at highest frequency; $\Delta \varepsilon_{n}$ is the magnitude of $n^{\text {th }}$ relaxation; $\omega$ is the angular frequency in radians per second; $\tau_{n}$ is the relaxation time of the $n^{\text {th }}$ relaxation; $\alpha_{n}$ is an empirical parameter that accounts for broadening of the $n^{\text {th }}$ relaxation; $\sigma_{s}$ is the static conductivity; and $\varepsilon_{0}$ is absolute permittivity. The measured dielectric properties were fitted to the two-pole ColeCole models for the cortex and medulla tissues of bovine adrenal gland described by the parameters in Table I.

\section{TABLE I}

Parameters of the two pole Cole-Cole model of bovine adrenal tissues [16]

\begin{tabular}{|c|c|c|}
\hline Parameter & Adrenal Cortex & Adrenal Medulla \\
\hline $\boldsymbol{\varepsilon}_{\infty}$ & 3.57 & 3.88 \\
\hline $\boldsymbol{\Delta} \varepsilon_{\boldsymbol{I}}$ & 47.08 & 52.95 \\
\hline $\boldsymbol{\tau}_{\boldsymbol{I}}$ & $8.33 \mathrm{ps}$ & $7.01 \mathrm{ps}$ \\
\hline $\boldsymbol{\alpha}_{\boldsymbol{I}}$ & 0.16 & 0.17 \\
\hline $\boldsymbol{\Delta} \varepsilon_{\boldsymbol{2}}$ & 52.31 & 62.05 \\
\hline $\boldsymbol{\tau}_{\boldsymbol{2}}$ & $1.69 \mathrm{~ns}$ & $4.28 \mathrm{~ns}$ \\
\hline $\boldsymbol{\alpha}_{\boldsymbol{2}}$ & 0.03 & 0.14 \\
\hline $\boldsymbol{\sigma}_{\boldsymbol{s}}$ & 0.46 & 0.62 \\
\hline
\end{tabular}

The sets of dielectric properties shown in Table I are slightly different, with the medulla being electromagnetically denser than the cortex. This contrast suggests that the microwave radiation will travel more rapidly in the cortex than in the medulla. In ablative treatments with short durations (less than a minute) direct heating due to microwave radiation will dominate conductive heating. Therefore, an improved dielectric characterisation of the targeted tissues allows more realistic numerical simulation of the temperature profiles [12].

The adrenal gland rests on the top portion of the kidney (see Figure 1). The lower surface of the adrenal gland is in direct contact with the kidney cortex tissue and its remaining free surfaces surrounded by fat. Figure 3 provides dielectric properties for the kidney cortex and fat tissue 
component in terms of permittivity and electric conductivity as reported in [19]. The thermal properties of the tissues are shown in Table II.

The numerical model generated for the multi-physics simulations discussed below does not include blood perfusion as this aspect is considered secondary for the size of the targeted region and the duration of the ablative treatment. Open boundary conditions were adopted to solve both the electromagnetic and thermal problems. Finally, the multilayer structure of the model was adaptively meshed to be denser in regions with higher permittivity. The size of meshing tetrahedrons spans between minimum and maximum edge lengths of $0.019 \mathrm{~mm}$ and $0.99 \mathrm{~mm}$, respectively.

It is proposed to position the ablation needle (applicator) at the interface between the fat and adrenal cortex layers, where the fat layer will act as an insulator due to significantly lower dielectric and thermal conductivity properties than the surrounding tissue. This placement of the needle should result in an ablation zone that primarily targets the cortex where hormone-eluding adenomas are located. The fat / cortex interface appears distinctly clear in CT imaging [20] and may provide a practical approach for targeting the cortex whilst sparing the medulla and preserving gland function.
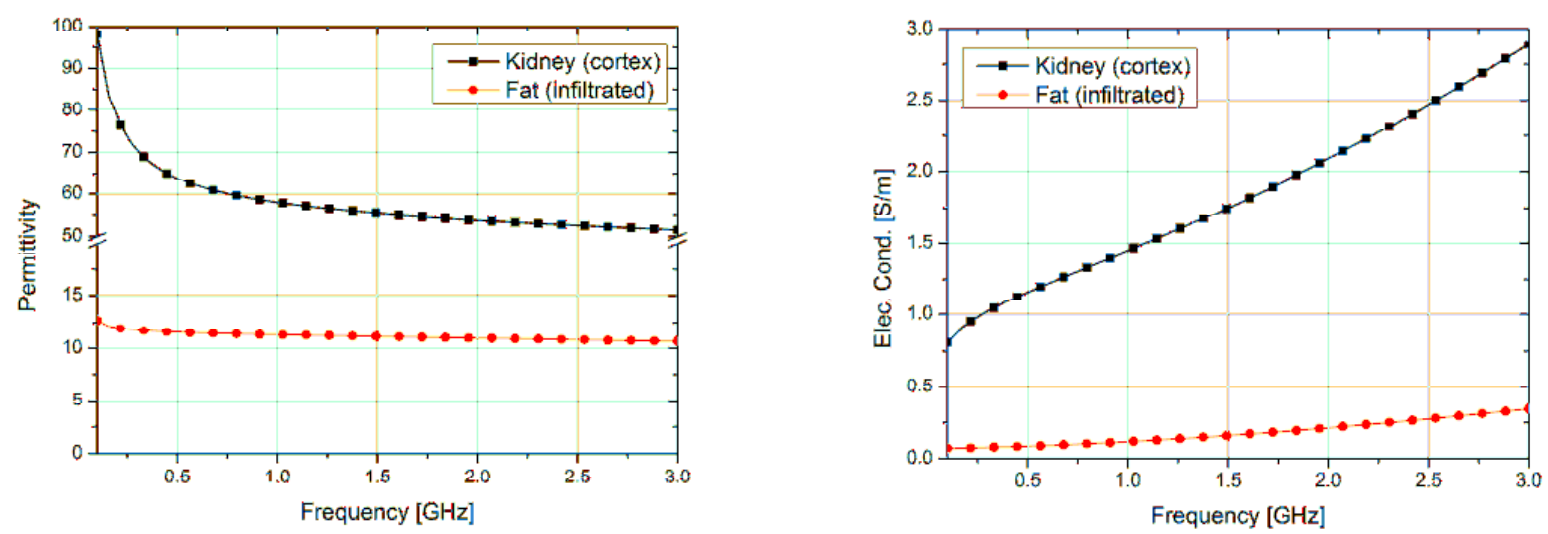

Figure 3: Permittivity and Electric conductivity of tissues adjacent to the adrenal site [19] 
Thermal properties of tissues in the adrenal site [19]

\begin{tabular}{|c|c|c|}
\hline Tissue & Heat capacity $\left[\mathbf{K j} / \mathbf{K g} /{ }^{\circ} \mathbf{C}\right]$ & $\begin{array}{c}\text { Thermal conductivity } \\
{\left[\mathbf{W} / \mathbf{m} /{ }^{\circ} \mathbf{C}\right]}\end{array}$ \\
\hline Adrenal medulla & 3.745 & 0.54 \\
\hline Adrenal cortex & 3.587 & 0.53 \\
\hline Infiltrated fat & 2.348 & 0.21 \\
\hline Kidney cortex & 3.763 & 0.53 \\
\hline
\end{tabular}

A numerical multilayer planar model was developed to validate this approach. It also sought to identify treatment durations and power settings where the ablation was confined by the fat layer to the cortex and did not reach the medulla. The ablation antenna was positioned parallel to the interface between the fat and the adrenal cortex layer in a simplified, planar geometry shown in Figure 4. The cortex and medulla were represented by two $5 \mathrm{~mm}$ thick layers corresponding a typical human adrenal gland structure. The fat capsule was modelled by a $15 \mathrm{~mm}$ thick layer, which is larger than that seen in vivo, but provided a simple and robust boundary condition for the preliminary analysis. The ablation antenna used in this paper is a 17 gauge omni-directional monopole applicator. The antenna is a coaxial monopole which is obtained by exposing a 6-mm length of the inner conductor of the feed cable when this is immerged in the water of the cooling system. The applicator presents an $\mathrm{S}_{11}$ performance better than $-12 \mathrm{~dB}$ in all working conditions under investigation. While more complex antenna solutions such as slot and directive antennas were explored, a simple coaxial monopole was adopted in this preliminary study to stress the attention on the complex multi-tissue model here proposed. 


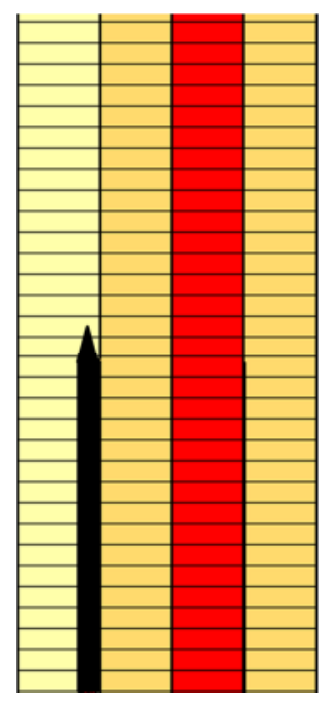

Figure 4: Planar multilayer model of the adrenal site

The planar model formed the basis for a more complex three dimensional (3D) simulation that included an anatomically realistic geometry. Figure 5 details the gland structure intended to represent a typical human adrenal. It was situated with the lower surfaces contacting the kidney cortex and the remainder surrounded by the fat capsule. The thickness of the adrenal cortex ranged from approximately 5 to $10 \mathrm{~mm}$. The ablation needle was positioned at the interface between the cortex and fat layer where the cortex is thinner as shown in Figure 5. The background medium in the model was set as blood to provide a stable boundary condition. The target ablation temperature was $60^{\circ} \mathrm{C}$ in all cases under investigation.

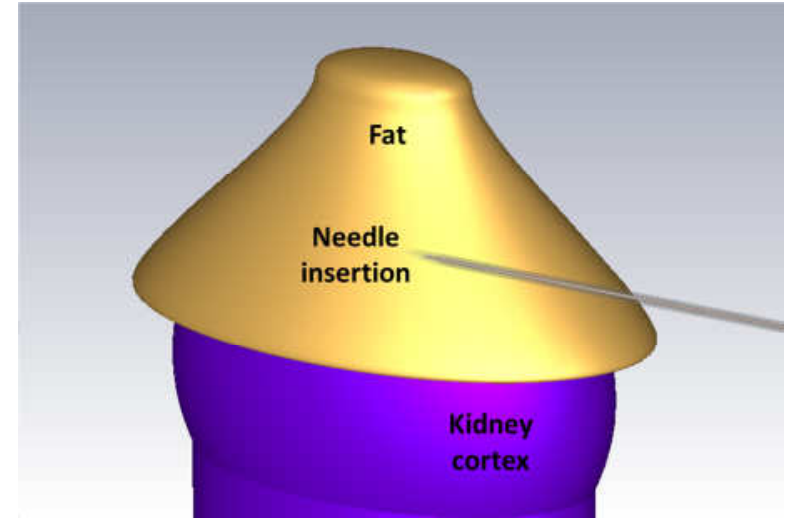

(a)

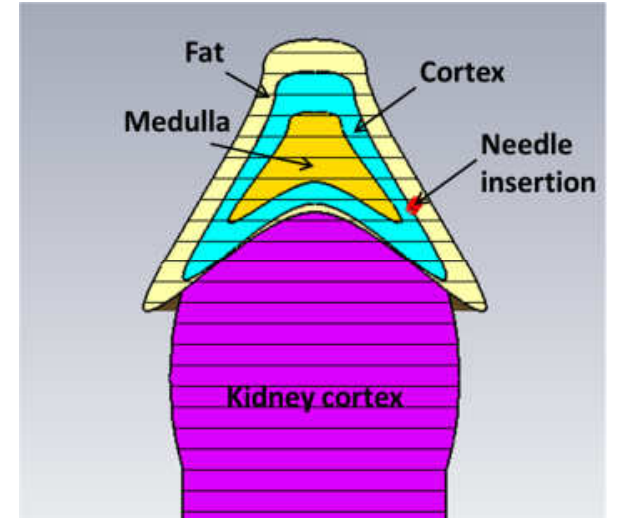

(b)

Figure 5: 3D multilayer model of the adrenal site and section view of the gland with needle inserted: (a) 3D view; (b) cross-section. 


\section{RESULTS}

This section details the results of the planar and 3D simulations. Three power levels (i.e. 30, 60 and $90 \mathrm{~W}$ delivered at the distal tip) and three treatment durations (i.e. 15, 30 and $45 \mathrm{~s}$ ) were considered.

Figure 6 displays the thermal distribution for each power and duration setting obtained from the planar model. As expected, limited interaction with the adipose layer was observed compared to the heat generated in the targeted cortex region at each power and time duration setting. The results also indicate that the ablation zone is confined to the cortex in the ablations at low duration ( $30 \mathrm{~s})$, even with high power. Direct heating will dominate at treatment with low durations, when there is limited time for conductive heating.

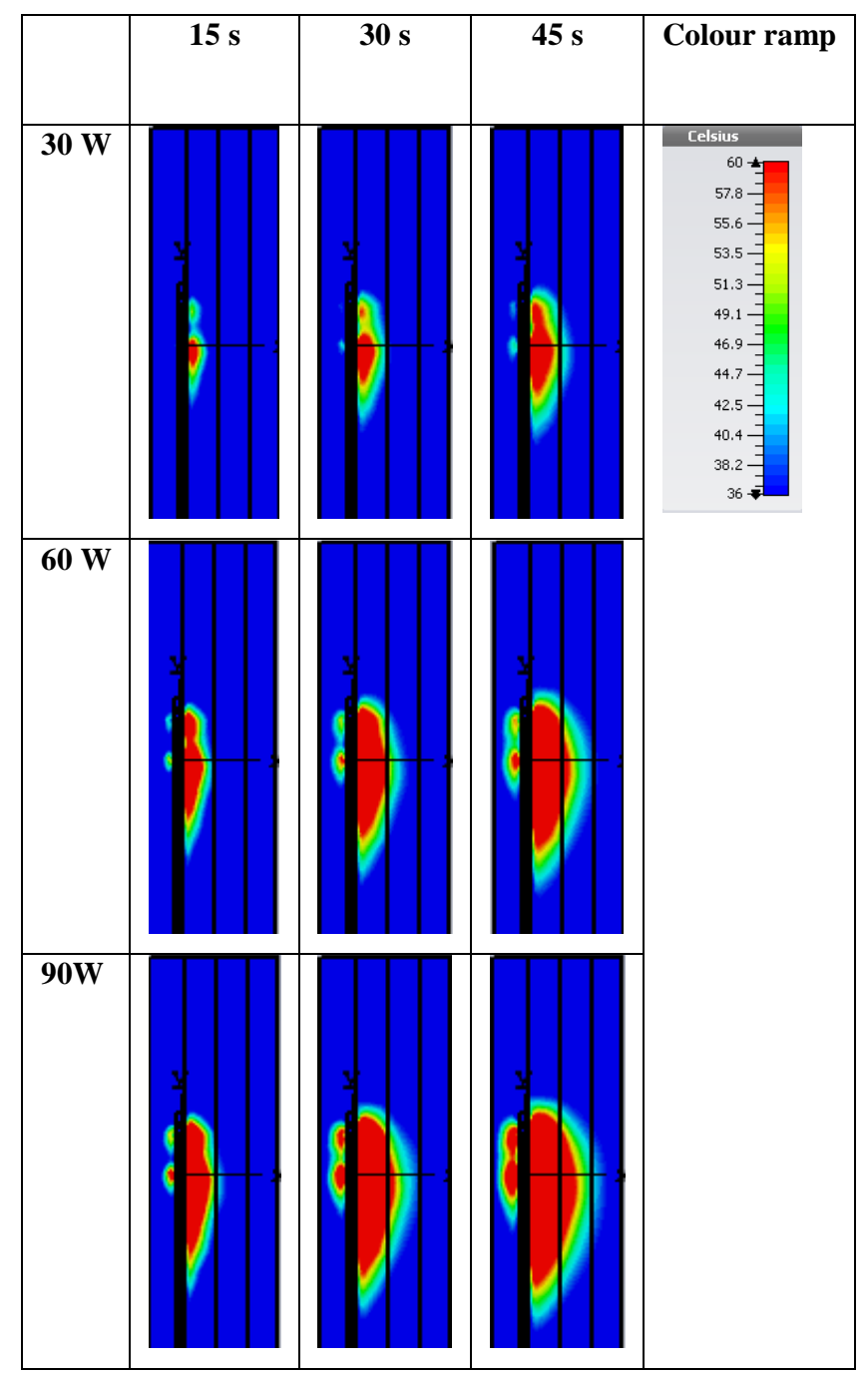

Figure 6: Ablation zone in planar multilayer model of the adrenal site for different power and time settings. 
Similar shielding effects by the fat capsule were observed in the results obtained from the simulation on the 3D geometry. The fat layer functioned to confine heating to the cortex at short treatment durations and low/medium input powers (see Figure 7). At all settings, the ablation zone is focussed towards the adrenal cortex and medulla; however at higher power and durations it extended beyond the thin fatty capsule, posing a risk of damage to surrounding structures (e.g. blood vessels, nerves, connective tissue, etc.). This demonstrates that the thickness and integrity of the fat capsule as well as accurate placement of the ablation probe is critical to maximising the shielding effect.

Analysis of the results shown in Figure 6 also indicate insufficient heating $\left(<60{ }^{\circ} \mathrm{C}\right)$ for tissue ablation occurred at $30 \mathrm{~W}$ input power, even at the high duration (45 s). Longer durations were not considered because they would favour conductive heating which does not differentiate between the tissue types as effectively as direct heating. The results of this analysis suggest that the optimum treatment may use a high power (e.g. 60 or $90 \mathrm{~W}$ ) for a short duration to maximise direct heating of the cortex whilst sparing the medulla.

The 3D model also provided a more realistic representation of the needle placement. Its location is complicated by the curvature of this fat / cortex interface. This resulted in discrepancies between the characteristics of the ablation zones observed from the planar model shown in Figure 6 where the needle was perfectly aligned to the fat/cortex interface. The heat maps of the 3D simulations are shown in Figure 7. In the 3D model the antenna is fully embedded into the fat tissue, which resulted in attenuation. This further highlights the importance of accurate probe placement to successfully target the cortex whilst sparing the surrounding tissues. 


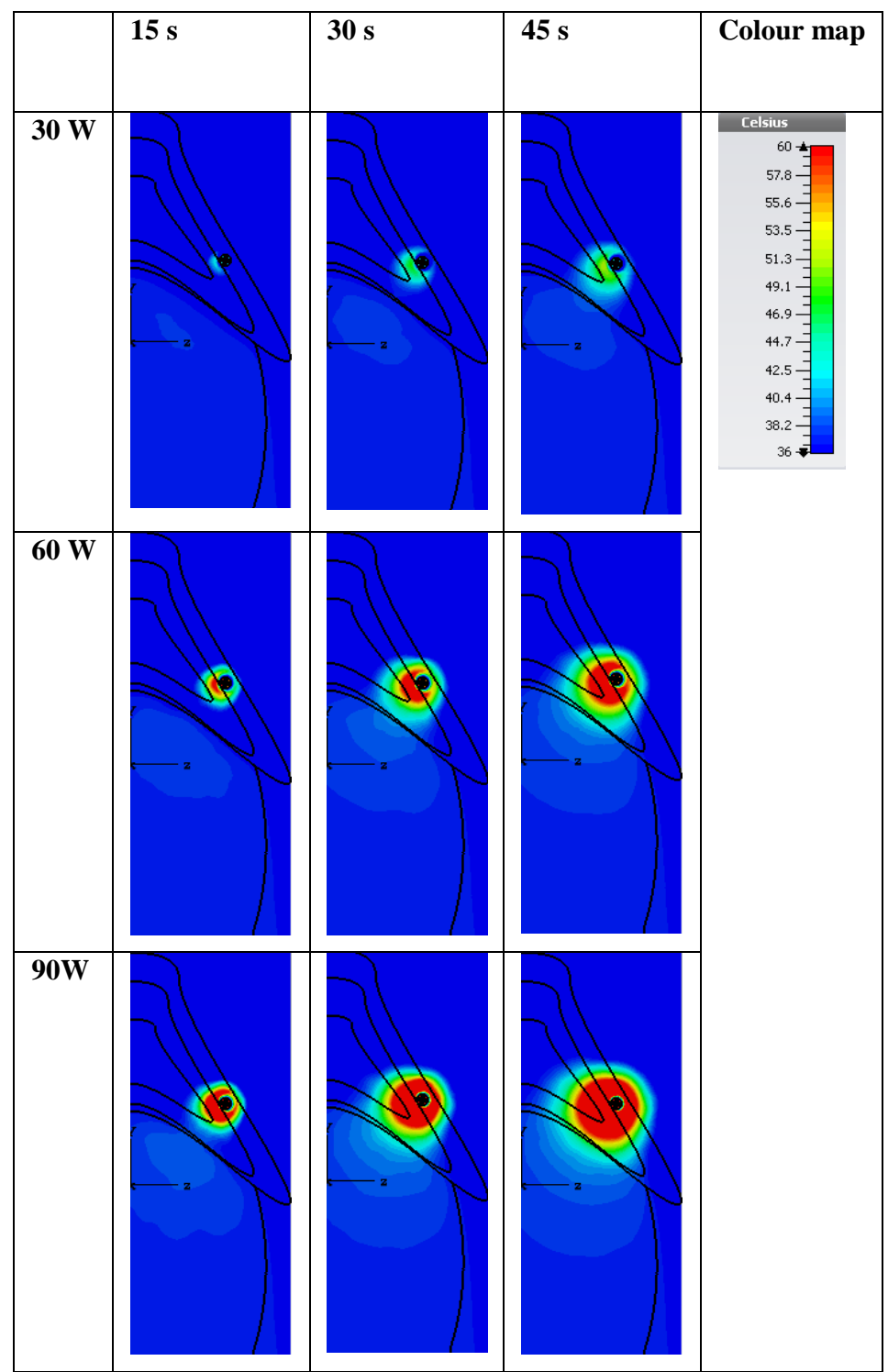

Figure 7: Ablation zone in 3D multilayer model of the adrenal site for different power/time settings.

The numerical analysis presented in the paper sought to carry out a realistic simulation of microwave ablation in the adrenal gland. However, the following limitations to the scope of the analysis are noted. Only one needle location and orientation was considered. Moreover, the properties of the hormone-eluding adenomas were not modelled as not yet fully characterized.

\section{CONCLUSIONS}

A multiphysics simulation of MWA in the adrenal gland including constituent tissues was presented. Planar and 3D models were used to analysis the effects of MW thermal ablation at 2.45 $\mathrm{GHz}$, performed with a 17 gauge omni-directional needle. It was demonstrated that placement of 
the needle at the interface between the cortex and fat capsule results in an ablation zone which targets the cortex. Furthermore, the use of high power with a short duration can maximise the direct heating in the targeted cortex of the gland. The results generated by this analysis suggest that treatments with a power of 60 or $90 \mathrm{~W}$ for $15 \mathrm{~s}$ result in ablation of a 5-mm thick adrenal cortex region while sparing surrounding medulla.

\section{Acknowledgement}

The research leading to these results has received funding from the European Research Council under the European Union's Horizon 2020 Programme/ERC PoC Grant REALTA n. 754308, and Irish Research Council New Foundations award. This paper has been supported by COST Action TD1301, MiMed.

\section{References}

[1] Alwan, A. Global status report on noncommunicable diseases 2010. (World Health Organization, 2015).

[2] WHO. Health 2020: A European policy framework and strategy for the 21st century. (World Health Organization, 2013).

[3] Rossi, G. P. et al. A Prospective Study of the Prevalence of Primary Aldosteronism in 1,125 Hypertensive Patients. J. Am. Coll. Cardiol. 48, 2293-2300 (2006).

[4] Aronova, A., Iii, T. J. F. \& Zarnegar, R. Management of hypertension in primary aldosteronism. World J. Cardiol. 6, 227-33 (2014).

[5] NIH state-of-the-science statement on management of the clinically inapparent adrenal mass ("incidentaloma") NIH Consens State Sci Statements. 2002;19:1-25.

[6] Rossi, G. P. Prevalence and diagnosis of primary aldosteronism. Curr. Hypertens. Rep. 12, 342-348 (2010).

[7] Young, W. F. Adrenal causes of hypertension: Pheochromocytoma and primary aldosteronism. Rev. Endocr. Metab. Disord. 8, 309-320 (2007).

[8] Brace, C. L. Thermal Tumor Ablation in Clinical Use. IEEE Pulse. 2011 Sep-Oct; 2(5): 2838.

[9] Brace, C. L. Radiofrequency and microwave ablation of the liver, lung, kidney and bone: What are the differences. Curr Probl Diagn Radiol 38, 135-143 (2009). 
[10] Wood, B. J., Abraham, J., Hvizda, J. L., Alexander, H. R., \& Fojo, T. (2003). Radiofrequency ablation of adrenal tumors and adrenocortical carcinoma metastases. Cancer, 97(3), 554-560.

[11] Yamakado, K. Image-guided ablation of adrenal lesions. Semin. Intervent. Radiol. 31, 149-156 (2014).

[12] Simon, C. J., Dupuy, D. E., \& Mayo-Smith, W. W. (2005). Microwave ablation: principles and applications. Radiographics, 25(suppl_1), S69-S83.

[13] Poulou, L. S., Botsa, E., Thanou, I., Ziakas, P. D. \& Thanos, L. Percutaneous microwave ablation vs radiofrequency ablation in the treatment of hepatocellular carcinoma. World J. Hepatol. 7, 1054-63 (2015).

[14] Mauri, G., Nicosia, L., Varano, G. M., Shyn, P., Sartori, S., Tombesi, P., Solbiati, L. (2017). Unusual tumour ablations: report of difficult and interesting cases. Ecancermedicalscience, 11, 733.

[15] Vincent, J. M., Morrison, I. D., Armstrong, P., \& Reznek, R. H. (1994). The size of normal adrenal glands on computed tomography. Clinical radiology, 49(7), 453-455.

[16] Shahzad, A., Clausing, D., Prakash, P., Dennedy, M. C., \& O'Halloran, M. (2017, September). Broadband dielectric properties of adrenal gland for accurate anatomical modelling in medical applications. In Electromagnetics in Advanced Applications (ICEAA), 2017 International Conference on (pp. 1465-1468). IEEE.

[17] Peyman A., and Gabriel C., "Dielectric properties of porcine glands, gonads and body fluids.", Physics in medicine and biology, vol. 57, no. 19, p. 339, 2012.

[18] C. Gabriel. Compilation of the Dielectric Properties of Body Tissues at RF and Microwave Frequencies, Report N.AL/OE-TR- 1996-0037, Occupational and environmental health directorate, Radiofrequency Radiation Division, Brooks Air Force Base, Texas (USA), 1996.

[19] Hasgall P.A., Di Gennaro F., Baumgartner C., Neufeld E., Gosselin M.C., Payne D., Klingenböck A., and Kuster N., "IT'IS Database for thermal and electromagnetic parameters of biological tissues," Version 3.0, September 01st, 2015.

[20] Blake M.A., Cronin C.G., Boland G.W., "Adrenal Imaging”, American Journal of Roentgenology 194:6, 1450-1460, 2010. 


\section{Biographies}

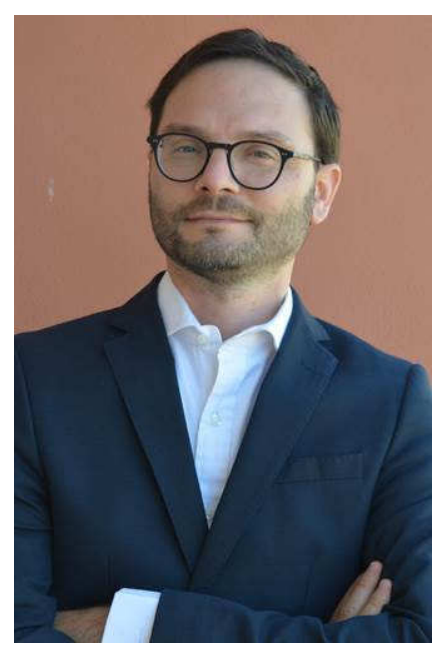

Giuseppe Ruvio completed a Specialist Master of Management in Clinical Engineering in 2017 from the University of Trieste, Italy, a Ph.D. on Microwave Engineering in 2009 from the Dublin Institute of Technology (DIT) and a Laurea degree from the University of Siena, Italy, in 2002. He recently joined the National University of Ireland Galway after research fellowships with DIT and the Trinity College University of Dublin. His recent research focuses on breast cancer microwave imaging, tissue-mimicking material engineering and dielectric properties measurement of biological tissues. He is currently lead electronic engineer in a commercialisation project on microwave ablation. Dr Ruvio is IEEE Senior member and he was awarded the Best Presentation Prize conferred by Bell Labs at the CTVR Conference 2012; the 2009 Hothouse Commercialisation Prize, the 2008 CST University Publication Award and the 2006 best paper prize at the Loughborough Antennas and Propagation Conference.

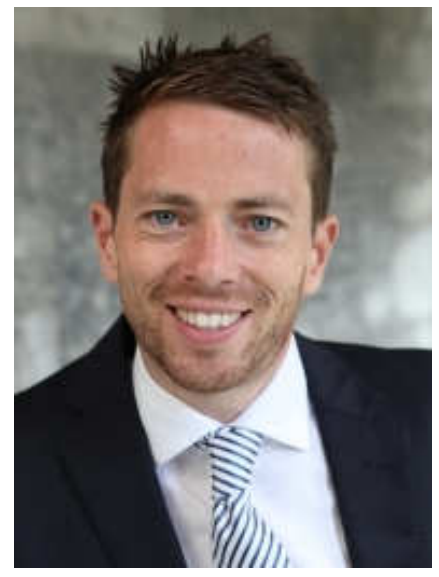

Jimmy Eaton-Evans is a mechanical design engineer with over 14 years of experience working in the field of medical device R\&D. After receiving his $\mathrm{PhD}$ from the University of Limerick in 2006, he completed postdoctoral research at the University of Oxford that focused on early stage medical device development. His industry experience includes working within both large multinational and small start-ups to bring new medical device solutions to market. He has published 21 peer review journal articles and is the named inventor on five patent filings.

In 2015 he completed the Bioinnovate Fellowship programme at NUIG in collaboration with the Mayo Clinic (MN, USA), which was focused on identifying new opportunities of innovation in gastroenterology. This provided the basis for him to secure funding of $€ 500 \mathrm{k}$ from Enterprise Ireland to commercialise a new, endoscopically delivered microwave ablation technology. $\mathrm{He}$ is currently leading the mechanical development of this device, on the journey to clinical use.

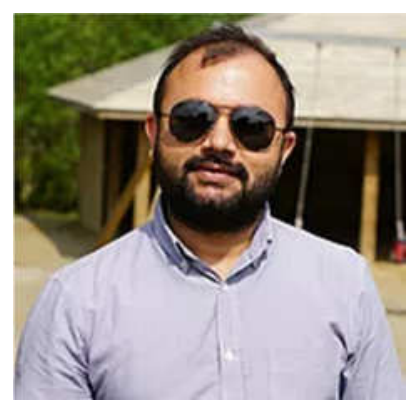

Atif Shahzad is a senior postdoctoral researcher and team lead ablation technologies at Translational Medical Device Lab, National University of Ireland, Galway. He received BSc (honors) in computer engineering from COMSATS Institute of Information Technology, Pakistan, and MSc in electronics engineering from University of Leeds, United Kingdom. He is associated with NUI Galway since 2011, starting $\mathrm{PhD}$ in microwave imaging for medical applications. His research is focused on thermal ablation and sensing technologies, medical signal and image processing, applied electromagnetic, computational modelling, and dielectric spectroscopy. 


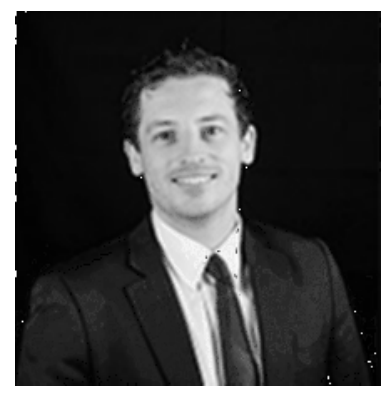

Martin O'Halloran is a European Research Council (ERC) and Science Foundation Ireland (SFI) Investigator at the National University of Ireland Galway. Reflecting the interdisciplinary nature of his research, he holds a joint affiliation with the College of Engineering and Informatics; and the College of Medicine, Nursing \& Health Sciences, and leads the Translational Medical Device Laboratory in the Lambe Institute. Dr O'Halloran has received over 30 national and international research awards, and was recently awarded SFI's Early-Stage Researcher of the Year, Engineers Ireland Chartered Engineer of the Year, and the European Research Council's Starting Investigator Grant. He was a co-proposer of a European COST Action (entitled "MiMED"), and is now leading a network of over 180 medical device researchers from 24 countries, focused on the clinical evaluation and commercialisation of novel medical devices in Europe. 